\title{
A Symmetric Function of Increasing Forests
}

\author{
Alex Abreu and Antonio Nigro \\ Instituto de Matemática e Estatística, Universidade Federal Fluminense, Rua Prof. M. W. de Freitas, S/N, 24210-201 Niterói, \\ Rio de Janeiro, Brasil; E-mail: alexbra1@gmail.com.
}

Received: 22 June 2020; Revised: 21 December 2020; Accepted: 2 April 2021

2020 Mathematics Subject Classification: Primary - 05A05; Secondary - 05E05

\begin{abstract}
For an indifference graph $G$, we define a symmetric function of increasing spanning forests of $G$. We prove that this symmetric function satisfies certain linear relations, which are also satisfied by the chromatic quasisymmetric function and unicellular LLT polynomials. As a consequence, we give a combinatorial interpretation of the coefficients of the LLT polynomial in the elementary basis (up to a factor of a power of $(q-1)$ ), strengthening the description given in [4].
\end{abstract}

\section{Contents}

1 Introduction 1

2 Preliminaries $\quad \mathbf{7}$

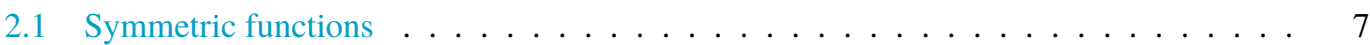

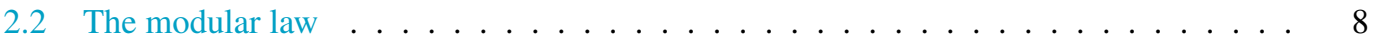

2.3 Permutations and increasing forests . . . . . . . . . . . . . . . 9

3 The symmetric function of increasing forests $\quad 11$

4 Relation with the chromatic quasisymmetric function and LLT polynomials 16

5 Chromatic quasisymmetric functions and LLT polynomials in other bases 18

\section{Introduction}

In 1730, Stirling introduced what are now called (signless) Stirling numbers of the first kind. These numbers $s(n, k)$ may be defined via the following equality:

$$
t(t-1) \cdots(t-n+1)=\sum_{k}(-1)^{n-k} s(n, k) t^{k} .
$$

One way to interpret this elementary identity is to notice that the left-hand side is the chromatic polynomial of the complete graph $K_{n}$ on $n$ vertices, so the numbers $s(n, k)$ are the coefficients of the chromatic polynomial in the basis $t^{k}$. A combinatorial interpretation for the $s(n, k)$ is the number of increasing forests with $k$ components and vertex set $[n]=\{1, \ldots, n\}$ (see [7]). An increasing tree $T$ is a tree with totally ordered vertex set such that the vertices along any path starting on the minimal vertex go in increasing order (see Figure 1). An increasing forest is a forest with totally ordered vertex set such that its components are increasing trees.

(C) The Author(s), 2021. Published by Cambridge University Press. This is an Open Access article, distributed under the terms of the Creative Commons Attribution licence (http://creativecommons.org/licenses/by/4.0/), which permits unrestricted re-use, distribution, and reproduction in any medium, provided the original work is properly cited. 

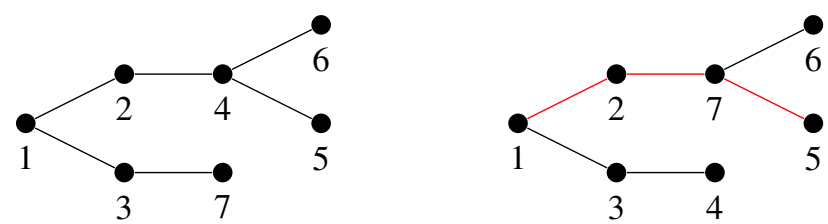

Figure 1. An increasing tree (left) and a non-increasing tree (right) with a non-increasing path highlighted.

With these definitions, we see that the coefficient of $t^{k}$ in the chromatic polynomial of $K_{n}$ counts (up to a sign) the number of increasing spanning forests of $K_{n}$ with $k$ components.

This interpretation actually holds for a larger class of graphs, namely, graphs that have a perfect elimination ordering. A perfect elimination ordering is an ordering $v_{1}, v_{2}, \ldots, v_{n}$ of the vertex set of $G$ such that, for each vertex $v_{j}$, the set $\left\{v_{i} ; i<j,\left\{v_{i}, v_{j}\right\} \in E(G)\right\}$ induces a clique of $G$. In this paper, we will assume that $G$ has vertex set $[n]$ and that the usual ordering of $[n]$ is a perfect elimination ordering. Then [14, Theorem 26] states that the coefficient of $t^{k}$ in $\chi_{G}(t)$ counts (up to a sign $\left.(-1)^{k}\right)$ the number of increasing spanning forests of $G$ with exactly $k$ components.

The chromatic polynomial of a graph admits a symmetric function generalization introduced by Stanley in [21]. Given a graph $G$, it is defined as

$$
\operatorname{csf}(G):=\sum_{\kappa} x_{\kappa}
$$

where the sum runs through all proper colorings of the vertices $\kappa: V(G) \rightarrow \mathbb{N}$ and $x_{\kappa}:=\prod_{v \in V(G)} x_{\kappa(v)}$. A coloring $\kappa$ is proper if $\kappa(v) \neq \kappa\left(v^{\prime}\right)$ whenever $v$ and $v^{\prime}$ are adjacent. We have that $\operatorname{csf}(G)$ lies in $\Lambda$, the algebra of symmetric functions.

The algebra $\Lambda$ has three important sets of generators called the elementary, power sum, and complete symmetric functions

$$
e_{n}(x)=\sum_{i_{1}<i_{2}<\ldots<i_{n}} x_{i_{1}} x_{i_{2}} \cdots x_{i_{n}}, \quad p_{n}(x)=\sum_{i} x_{i}^{n}, \quad h_{n}(x)=\sum_{i_{1} \leq i_{2} \leq \ldots \leq i_{n}} x_{i_{1}} x_{i_{2}} \cdots x_{i_{n}},
$$

where $x$ denotes the collection of variables $\left(x_{1}, x_{2}, \ldots\right)$.

Every element in $\Lambda$ can be written as a polynomial in one of the generators above. Moreover, for every partition $\lambda=\left(\lambda_{1}, \ldots, \lambda_{\ell(\lambda)}\right)$, we define $e_{\lambda}(x):=\prod e_{\lambda_{i}}(x), p_{\lambda}(x):=\prod p_{\lambda_{i}}(x)$ and $h_{\lambda}(x):=\prod h_{\lambda_{i}}(x)$. Each of the sets $\left\{e_{\lambda}(x)\right\},\left\{p_{\lambda}(x)\right\}$ and $\left\{h_{\lambda}(x)\right\}$ is a homogeneous basis for $\Lambda$.

If we set $x_{1}=x_{2}=\ldots=x_{m}=1$ and $x_{m+1}=x_{m+2}=\ldots=0$, then $\operatorname{csf}(G)(1, \ldots, 1,0, \ldots)$ is precisely the number of ways to color $G$ with $m$ colors, which is simply $\chi_{G}(m)$. Moreover, via this substitution we have that

$$
e_{n}(1, \ldots, 1,0, \ldots)=\left(\begin{array}{c}
m \\
n
\end{array}\right), h_{n}(1, \ldots, 1,0, \ldots)=\left(\begin{array}{c}
m+n-1 \\
n
\end{array}\right),
$$

and $p_{n}(1, \ldots, 1,0, \ldots)=m$. We can actually make $m$ into an indeterminate $t$. If we define the specialization map $\epsilon: \Lambda \rightarrow \mathbb{Q}[t]$ as the homomorphism of algebras given by $\epsilon\left(p_{n}\right)=t$ for every $n=1,2, \ldots$, then we have that $\epsilon\left(e_{n}\right)=t(t-1) \cdots(t-n+1) / n !, \epsilon\left(h_{n}\right)=t(t+1) \cdots(t+n-1) / n !$, and, as expected, $\epsilon(\operatorname{csf}(G))=\chi_{G}(t)$. We refer to [17, Example 1, page 26] for more details about the specialization map.

Via the specialization map, we have $\epsilon\left(p_{\lambda}\right)=t^{\ell(\lambda)}$ for every partition $\lambda$. So, the analogue of writing $\chi_{G}$ in the basis $t^{k}$ is to express $\operatorname{csf}(G)$ in the basis $p_{\lambda}$ with $\lambda \vdash n$. For instance, if $G$ is the graph in Figure 2, we have that

$$
\operatorname{csf}(G)=p_{1,1,1,1}-4 p_{2,1,1}+p_{2,2}+4 p_{3,1}-2 p_{4} .
$$




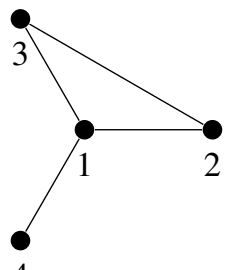

4

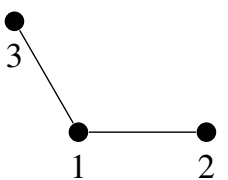

4

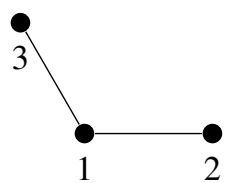

4
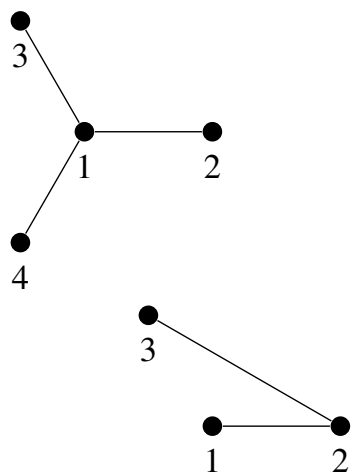

4

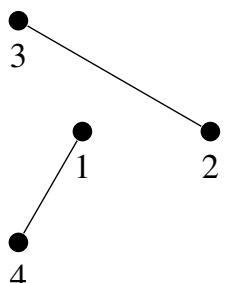

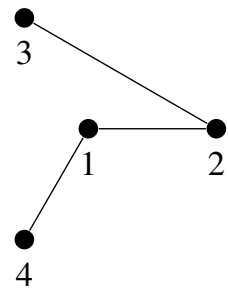

3

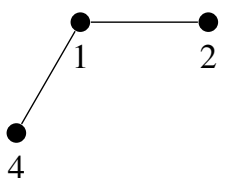

Figure 2. A graph $G$ with a perfect elimination ordering and chromatic polynomial $t^{4}-4 t^{3}+5 t^{2}-2 t$, its 2 increasing spanning forests with 1 component and its 5 increasing spanning forests with 2 components.

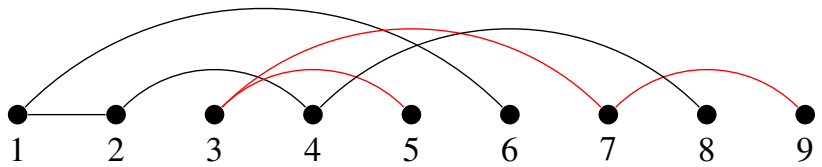

Figure 3. The increasing forest associated with the permutation $\sigma=(16248)(3795)$.

The attentive reader may notice that the five increasing spanning forests of $G$ with two components are nicely divided into two groups: there are four forests with components of either 3 vertices or 1 vertex, and one forest with components having 2 vertices. With that in mind, we define the partition $\lambda(F)$ of a forest $F$ as the partition induced by the number of vertices of the components of $F$.

Going back to the complete graph, the chromatic symmetric function is $n ! e_{n}(x)$. In that case, we have the well-known Newton identity

$$
n ! e_{n}=\sum_{\lambda \vdash n}(-1)^{n-\ell(\lambda)} a_{\lambda} p_{\lambda}(x)
$$

where $a_{\lambda}$ is the number of permutations in $n$ elements with cycle partition $\lambda$. The number $a_{\lambda}$ also counts increasing forests with vertex set $[n]$ and partition $\lambda$. Indeed, we can construct a bijection between permutations with size $n$ and increasing forests with vertex set $[n]$ as follows (see [22, Example 1.3.15]). For each cycle $\tau=\left(j_{1}, j_{2}, \ldots, j_{k}\right)$, with $j_{1} \leq j_{l}$ for every $l \in[k]$, we construct an increasing tree $T_{\tau}$ with vertex set $\left\{j_{1}, \ldots, j_{k}\right\}$, where $j_{i}$ is connected to the rightmost element $j_{l}$ of $\tau$ that precedes $j_{i}$ and that is less than $j_{i}$. If $\sigma$ is a permutation in $S_{n}$, write $\sigma$ in cyclic notation $\sigma=\tau_{1} \ldots \tau_{k}$ and define the forest $F_{\sigma}$ as $T_{\tau_{1}} \cup T_{\tau_{2}} \cup \ldots \cup T_{\tau_{k}}$. It follows from the construction that if $\sigma$ has cycle partition $\lambda$, then $\lambda\left(F_{\sigma}\right)=\lambda$ as well. See Figure 3 for an example. 
Actually, if $G$ is a graph with a perfect elimination ordering, then

$$
\operatorname{csf}(G)=\sum_{F}(-1)^{n-\ell(\lambda(F))} p_{\lambda(F)}(x),
$$

where the sum runs through all increasing spanning forests of $G{ }^{1}$

The Stirling numbers of the first kind admit a $q$-deformation $s_{q}(n, k)$ introduced by Gould [13], defined through the identity

$$
t\left(t-[1]_{q}\right)\left(t-[2]_{q}\right) \cdots\left(t-[n-1]_{q}\right)=\sum_{k}(-1)^{n-k} s_{q}(n, k) t^{k},
$$

where $[j]_{q}=\left(q^{j}-1\right) /(q-1)$. Since $s(n, k)$ enumerates increasing forests, we could ask what statistic on increasing forests gives $s_{q}(n, k)$. In another direction, we could ask what is a $q$-analogue of Equation (2). Of course, we would first have to modify either $e_{n}(x)$ or $p_{\lambda}(x)$.

To simplify matters and avoid unnecessary signs, we will apply the usual involution $\omega$ to Equation (2). Recall that $\omega: \Lambda \rightarrow \Lambda$ is defined by $\omega\left(e_{n}(x)\right):=h_{n}(x)$ and satisfies $\omega\left(p_{n}(x)\right)=(-1)^{n-1} p_{n}(x)$. Then equation (2) becomes an additional Newton identity:

$$
n ! h_{n}(x)=\sum_{\lambda \vdash n} a_{\lambda} p_{\lambda}(x) .
$$

Another relation involving the power sum and complete symmetric functions is the recursion

$$
n h_{n}(x)=\sum_{i=1}^{n} h_{n-i}(x) p_{i}(x) .
$$

With this in mind, we define the symmetric functions $\rho_{n}(x ; q)$ in $\Lambda_{q}:=\Lambda[q]$ by the recursion

$$
[n]_{q} h_{n}(x)=\sum_{i=1}^{n} h_{n-i}(x) \rho_{i}(x ; q) .
$$

For instance, we have that

$$
\begin{aligned}
& \rho_{1}(x ; q)=h_{1}(x), \\
& \rho_{2}(x ; q)=(q+1) h_{2}(x)-h_{1,1}(x), \\
& \rho_{3}(x ; q)=\left(q^{2}+q+1\right) h_{3}(x)-(q+2) h_{2,1}(x)+h_{1,1,1}(x) .
\end{aligned}
$$

We note that $\rho_{n}(x ; q)$ interpolates between the power sum and the elementary symmetric function, in the sense that $\rho_{n}(x ; 1)=p_{n}(x)$ and $\rho_{n}(x ; 0)=(-1)^{n-1} e_{n}(x)$. The reader familiar with symmetric functions will quickly realize that $\rho_{n}(x ; q)$ is actually a modification of the Hall-Littlewood polynomial $P_{n}(x ; q)$, namely $\rho_{n}(x ; q)=q^{n-1} P_{n}\left(x ; q^{-1}\right)$. Defining $\rho_{\lambda}(x ; q):=\prod \rho_{\lambda_{i}}(x ; q)$, for example

$$
\begin{aligned}
\rho_{3,2}(x ; q)= & \left(q^{3}+2 q^{2}+2 q+1\right) h_{3,2}(x)-\left(q^{2}+q+1\right) h_{3,1,1}(x) \\
& -\left(q^{2}+3 q+2\right) h_{2,2,1}(x)+(2 q+3) h_{2,1,1,1,1}(x)-h_{1,1,1,1,1}(x),
\end{aligned}
$$

we get a $q$-analogue of Equation (4),

$$
n !_{q} h_{n}(x)=\sum_{\lambda \vdash n} a_{\lambda}(q) \rho_{\lambda}(x ; q)
$$

${ }^{1}$ The authors could not find this precise statement in the literature, but it readily follows from [21, Corollary 2.7] and [14]. 


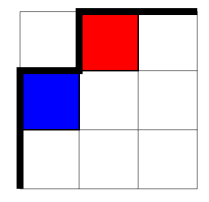

$h=(2,3,3)$

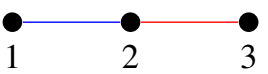

$G$

Figure 4. A Hessenberg function $h$ and its associated indifference graph.

where $n !_{q}:=\prod_{j=1}^{n}[j]_{q}$ and $a_{\lambda}(q)$ is a polynomial with non-negative integer coefficients. Moreover, we have that $s_{q}(n, k)=\sum a_{\lambda}(q)$, where the sum runs through all partitions $\lambda$ of $n$ with length $\ell(\lambda)=k$ (see Proposition 2.1 and Corollary 3.7).

Since $s(n, k)$ and $a_{\lambda}$ enumerate increasing forests, it is natural to ask if there is a statistic on increasing forests that gives $s_{q}(n, k)$ and $a_{\lambda}(q)$, and more generally if there is a $q$-analogue of Equation (3).

One of the main goals of this paper is to answer this question. We first notice that the left-hand side of Equation (3) already has a $q$-analogue introduced in [20], called the chromatic quasisymmetric function of $G$. For a graph $G$ with set of vertices $[n]$, the chromatic quasisymmetric function $\operatorname{csf}_{q}(G)$ is defined as

$$
\operatorname{csf}_{q}(G):=\sum_{\kappa} q^{\operatorname{asc}_{G}(k)} x_{\kappa}
$$

where the sum runs through all proper colorings of $G$ and

$$
\operatorname{asc}_{G}(\kappa):=|\{(i, j) ; i<j, \kappa(i)<\kappa(j) ;\{i, j\} \in E(G)\}|
$$

is the number of ascents of the coloring $\kappa$.

For the right-hand side of Equation (3), $\rho_{\lambda}(x ; q)$ will play the part of $p_{\lambda}(x)$, so it remains to find a statistic $\mathrm{wt}_{G}(F)$ for every increasing spanning forest $F$ of $G$ such that

$$
\omega\left(\operatorname{csf}_{q}(G)\right)=\sum_{F} q^{\mathrm{wt}_{G}(F)} \rho_{\lambda(F)}(x ; q) .
$$

One complication is that $\operatorname{csf}_{q}(G)$ is not usually symmetric, in which case there is no chance for the equality above to hold. To avoid this, we will restrict our attention in the rest of this paper to a class of graphs for which $\operatorname{csf}_{q}(G)$ is known to be symmetric, namely indifference graphs.

A graph is called an indifference graph if its vertex set is $[n]:=\{1,2, \ldots, n\}$ for some natural $n$ and such that if $\{i, j\}$ is an edge with $i<j$, then $\{i, k\}$ and $\{k, j\}$ are also edges for every $k$ such that $i<k<j$. In particular, on every indifference graph, the usual ordering of $[n]$ is a perfect elimination ordering.

Indifference graphs are naturally associated with Hessenberg functions. A Hessenberg function is a non-decreasing function $\mathbf{m}:[n] \rightarrow[n]$ such that $\mathbf{m}(i) \geq i$ for every $i \in[n]$. The graph $G_{\mathbf{m}}$ induced by $\mathbf{m}$ is the graph with vertex set $[n]$ and edge set $\{(i, j) ; i<j \leq \mathbf{m}(i)\}$. Every indifference graph arises in this way.

For a permutation $\sigma \in S_{n}$, we say that $\sigma \leq \mathbf{m}$ if $\sigma(i) \leq \mathbf{m}(i)$ for every $i \in[n]$, and we denote by $S_{n, \mathbf{m}}$ the set of all permutations $\sigma \in S_{n}$ such that $\sigma \leq \mathbf{m}$. One easy observation is that $\sigma \leq \mathbf{m}$ if and only if $F_{\sigma}$ is a increasing spanning forest of $G_{\mathbf{m}}$. Equation (3) then becomes

$$
\omega(\operatorname{csf}(G))=\sum_{\sigma \leq \mathbf{m}} p_{\lambda(\sigma)}(x) .
$$

This was known ante litteram in the work of Stanley and Stembridge [23]. We mention this as it is easier to define the statistic $\mathrm{wt}_{G}(F)$ in terms of the associated permutation. 
A $G$-inversion of $\sigma$ is a pair $(i, j)$ with $i<j, \sigma(i)>\sigma(j)$, and $\{\sigma(j), \sigma(i)\} \in E(G)$. Also, we let $\sigma^{c}$ be the permutation obtained by removing the parentheses in the cyclic notation of $\sigma$. Then we define $\mathrm{wt}_{G}(\sigma)$ to be the number of $G$-inversions of $\sigma^{c}$.

Definition 1.1. Let $\mathbf{m}:[n] \rightarrow[n]$ be a Hessenberg function with associated indifference graph $G$. For each partition $\lambda \vdash n$, we define

$$
c_{\lambda}(\mathbf{m}, q)=c_{\lambda}(G, q):=\sum_{\substack{\sigma \leq \mathbf{m} \\ \lambda(\sigma)=\lambda}} q^{\mathrm{wt}_{G}(\sigma)} .
$$

We have the following theorem.

Theorem 1.2. If $G$ is an indifference graph, then

$$
\omega\left(\operatorname{csf}_{q}(G)\right)=\sum_{\sigma \leq \mathbf{m}} q^{\mathrm{wt}_{G}(\sigma)} \rho_{\lambda(\sigma)}(x ; q)=\sum_{\lambda \vdash n} c_{\lambda}(G, q) \rho_{\lambda}(x ; q) .
$$

Increasing forests also play a role in another symmetric function related to colorings of $G$. If $G$ is an indifference graph, the unicellular LLT polynomial associated with $G$ is defined as

$$
\operatorname{LLT}(G):=\sum_{\kappa} q^{\operatorname{asc}_{G}(\kappa)} x_{\kappa}
$$

where the sum runs through all (not necessarily proper) colorings of $G .^{2}$

Since $\operatorname{csf}_{q}$ and the unicellular LLT polynomial are closely related by a plethystic transformation (see [8]), we also get

Theorem 1.3. If $G$ is an indifference graph, then

$$
\operatorname{LLT}(G)=\sum_{\sigma \leq \mathbf{m}}(q-1)^{n-\ell(\lambda(\sigma))} q^{\mathrm{wt}_{G}(\sigma)} e_{\lambda(\sigma)}(x) .
$$

In particular, we have that

$$
\operatorname{LLT}(G)=\sum_{\lambda \vdash n}(q-1)^{n-\ell(\lambda)} c_{\lambda}(G, q) e_{\lambda}(x) .
$$

As a direct corollary, we obtain

Corollary 1.4. When $G$ is an indifference graph, we have that $\operatorname{LLT}(G ; q+1)$ is e-positive.

The $e$-positivity of the vertical strip LLT polynomials, after the shift $q \rightarrow q+1$, was first conjectured in [2] and [10], following [6] where several symmetric functions were conjectured to be $e$-positive after this shift. This conjecture has been recently proved in [9, Corollary 5.7] and [4, Corollary 2.10]. The latter paper also proves the explicit combinatorial description conjectured in [2]. This description also follows from Theorem 1.3 (see Proposition 4.1).

We note that Theorem 1.3 is a slight strengthening (in the unicellular case) of this conjecture, in the sense that we isolate the explicit contribution of the $(q-1)$ factors. Moreover, with a more careful examination of Theorem 1.3, we can actually extend Corollary 1.4 to vertical strip LLT polynomials (see Proposition 4.2).

The main idea to prove these results is to use the fact that $\operatorname{csf}_{q}$ and LLT are completely determined by certain linear relations and their values at the complete graphs, as proved in [1]. Consequently, it is enough to prove that the right-hand sides of Equations (5) and (6) also satisfy these relations.

${ }^{2}$ These polynomials can actually be defined in a more general setting; see [16]. 


\section{Preliminaries}

\subsection{Symmetric functions}

Let $\Lambda$ be the algebra of symmetric functions, and $\Lambda_{q}:=\Lambda[q]$. As usual, we let $[j]_{q}=\left(q^{j}-1\right) /(q-1)$ and $j !_{q}=\prod_{i=1}^{j}[i]_{q}$. We denote by $e_{\lambda}(x), h_{\lambda}(x), s_{\lambda}(x)$, and $p_{\lambda}(x)$ the elementary, complete, Schur, and power sum symmetric functions. Also, we let $P_{\lambda}(x ; q) \in \Lambda_{q}$ be the Hall-Littlewood polynomial (see [17, Chapter 3]). For our purposes, the relevant identity is

$$
P_{n}(x ; q)=\sum_{r=0}^{n-1}(-q)^{r} s_{n-r, 1^{r}}(x)
$$

(see [17, page 214]). Then we define $\rho_{n}(x ; q):=q^{n-1} P_{n}\left(x ; q^{-1}\right)$; alternatively, we could define $\rho_{n}:=$ $(-1)^{n-1} \omega\left(P_{n}(x ; q)\right)$. As usual, we set $\rho_{\lambda}(x ; q):=\prod \rho_{\lambda_{i}}(x ; q)$. Notice that $\rho_{\lambda}(x ; q)$ is, in general, not the same as $q^{|\lambda|-\ell(\lambda)} P_{\lambda}\left(x ; q^{-1}\right)$. We have that $\rho_{\lambda}(x ; q)$ interpolates between the power sum, $\rho_{\lambda}(x ; 1)=$ $p_{\lambda}(x)$, and the elementary symmetric functions, $\rho_{\lambda}(x ; 0)=(-1)^{n-\ell(\lambda)} e_{\lambda}(x)$.

Before stating a proposition that relates $\rho_{n}(x ; q)$ with the complete homogeneous symmetric functions $h_{n}(x)$, we make a few definitions. A domino is a connected horizontal strip, and a domino tabloid of shape $\lambda$ and type $\mu$ is a filling of the Young diagram of $\lambda$ with dominoes of length $\mu_{i}$, where dominoes of the same length are indistinguishable. The $q$-weight of a domino tabloid is the product $\prod\left[l_{i}\right]_{q}$, where $l_{i}$ is the length of the leftmost domino in the $i$-th row of the Young diagram of $\lambda$. We denote by $w_{\lambda \mu}(q)$ the sum of the $q$-weights of all domino tabloids of shape $\lambda$ and type $\mu$. Also, as usual, we define $z_{\lambda}:=\frac{n !}{a_{\lambda}}$, where $a_{\lambda}$ is the number of permutations with cycle partition $\lambda$.

Proposition 2.1. We have the following equalities:

1.

$$
[n]_{q} h_{n}(x)=\sum_{j=1}^{n} h_{n-j}(x) \rho_{j}(x ; q)
$$

2.

$$
n !_{q} h_{n}(x)=\left|\begin{array}{cccccc}
\rho_{1}(x ; q) & \rho_{2}(x ; q) & \rho_{3}(x ; q) & \cdots & \rho_{n-1}(x ; q) & \rho_{n}(x ; q) \\
-1 & \rho_{1}(x ; q) & \rho_{2}(x ; q) & \cdots & \rho_{n-2}(x ; q) & \rho_{n-1}(x ; q) \\
0 & -[2]_{q} & \rho_{1}(x ; q) & \cdots & \rho_{n-3}(x ; q) & \rho_{n-2}(x ; q) \\
0 & 0 & -[3]_{q} & \cdots & \rho_{n-4}(x ; q) & \rho_{n-3}(x ; q) \\
\vdots & \vdots & \vdots & \ddots & \vdots & \vdots \\
0 & 0 & 0 & \cdots & -[n-1]_{q} & \rho_{1}(x ; q)
\end{array}\right|
$$

3.

$$
\rho_{n}(x ; q)=\left|\begin{array}{cccccc}
h_{1}(x) & {[2]_{q} h_{2}(x)} & {[3]_{q} h_{3}(x)} & \cdots & {[n-1]_{q} h_{n-1}(x)} & {[n]_{q} h_{n}(x)} \\
1 & h_{1}(x) & h_{2}(x) & \cdots & h_{n-2}(x) & h_{n-1}(x) \\
0 & 1 & h_{1}(x) & \cdots & h_{n-3}(x) & h_{n-2}(x) \\
0 & 0 & 1 & \cdots & h_{n-4}(x) & h_{n-3}(x) \\
\vdots & \vdots & \vdots & \ddots & \vdots & \vdots \\
0 & 0 & 0 & \cdots & 1 & h_{1}(x)
\end{array}\right|
$$

4.

$$
\rho_{n}(x ; q)=\sum_{\alpha \models n}(-1)^{\ell(\alpha)-1}\left[\alpha_{1}\right]_{q} h_{\lambda(\alpha)}(x)
$$

5.

$$
\rho_{\lambda}(x ; q)=\sum_{\mu \vdash n}(-1)^{\ell(\lambda)-\ell(\mu)} w_{\lambda \mu}(q) h_{\mu}(x)
$$


6.

$$
\rho_{\lambda}(x ; q)=\frac{h_{\lambda}[(q-1) x]}{(q-1)^{\ell(\lambda)}} .
$$

7.

$$
\rho_{n}(x ; q)=\sum_{\lambda \vdash n} \frac{p_{\lambda}(x)}{z_{\lambda}}(q-1)^{\ell(\lambda)-1} \prod_{i=1}^{\ell(\lambda)}\left[\lambda_{i}\right]_{q}
$$

Proof. The first equality comes from Equation (8), the fact that $h_{n}=s_{n}$, and the Pieri formula,

$$
s_{n-j}(x) \cdot s_{j-r, 1^{r}}(x)=\sum_{a=0}^{\min (n-j, j-r-1)} s_{n-r-a, 1+a, 1^{r-1}}(x)+\sum_{a=0}^{\min (n-j-1, j-r-1)} s_{n-r-a-1,1+a, 1^{r}}(x) .
$$

Items (2), (3) are a consequence of item (1) and the expansion of the determinant. Items (4) and (5) follow from item (1), just adapting a standard argument [17, page 109].

To prove item (6), we define $q_{n}(x ; q)=(1-q) P_{n}(x ; q)$ as in [17, Page 209]. This means $\rho_{n}(x ; q)=$ $\frac{q^{n}}{q-1} q_{n}\left(x ; q^{-1}\right)$. By [19, Theorem 4.13, items (b) and (c)], we have that $\rho_{n}(x ; q)=\frac{q^{r}}{q-1} h_{n}\left[\left(1-q^{-1}\right) x\right]$, and hence $\rho_{n}(x ; q)=\frac{h_{n}[(q-1) x]}{q-1}$. Item (7) follows from [19, Theorem 4.13, item (d)].

\subsection{The modular law}

The chromatic symmetric function and the unicellular LLT polynomial satisfy a set of recurrences that characterizes them. We denote by $\mathcal{D}$ the set of Hessenberg functions, which we identify with the set of indifference graphs. We recall that $\mathcal{D}$ has a multiplication given by $G_{1} \cdot G_{2}=G_{1} \cup G_{2}$, where $G_{1} \cup G_{2}$ is the ordered union of $G_{1}$ and $G_{2}$.

Definition 2.2. We say that a function $f: \mathcal{D} \rightarrow \mathcal{A}$ satisfies the modular law if

$$
(1+q) f\left(\mathbf{m}_{1}\right)=q f\left(\mathbf{m}_{0}\right)+f\left(\mathbf{m}_{2}\right)
$$

whenever one of the following conditions holds:

1. There exists $i \in[n-1]$ such that $\mathbf{m}_{1}(i-1)<\mathbf{m}_{1}(i)<\mathbf{m}_{1}(i+1)$ and $\mathbf{m}_{1}\left(\mathbf{m}_{1}(i)\right)=\mathbf{m}_{1}\left(\mathbf{m}_{1}(i)+1\right)$ or $\mathbf{m}_{1}(i)=n$. Moreover, $\mathbf{m}_{0}$ and $\mathbf{m}_{2}$ satisfy $\mathbf{m}_{k}(j):=\mathbf{m}_{1}(j)$ for every $j \neq i$ and $k=0,2$, while $\mathbf{m}_{k}(i)=\mathbf{m}_{1}(i)-1+k$.

2. There exists $i \in[n-1]$ such that $\mathbf{m}_{1}(i+1)=\mathbf{m}_{1}(i)+1$ and $\mathbf{m}_{1}^{-1}(i)=\emptyset$. Moreover, $\mathbf{m}_{0}$ and $\mathbf{m}_{2}$ satisfy $\mathbf{m}_{k}(j):=\mathbf{m}_{1}(j)$ for every $j \neq i, i+1$ and $k=0,2$, while $\mathbf{m}_{0}(i)=\mathbf{m}_{0}(i+1)=\mathbf{m}_{1}(i)$ and $\mathbf{m}_{2}(i)=\mathbf{m}_{2}(i+1)=\mathbf{m}_{1}(i+1)$.

We have the following Theorems (see [1, Theorem 1.2]).

Theorem 2.3. The chromatic quasisymmetric function is the unique multiplicative function $\mathrm{csf}_{q}: \mathcal{D} \rightarrow$ $\Lambda_{q}$ satisfying the modular law such that $\operatorname{csf}_{q}\left(K_{n}\right)=n !_{q} e_{n}(x)$.

Theorem 2.4. The unicellular LLT polynomial is the unique multiplicative function LLT: $\mathcal{D} \rightarrow \Lambda_{q}$ satisfying the modular law such that

$$
\operatorname{LLT}\left(K_{n}\right)=\sum_{i=1}^{n} \operatorname{LLT}\left(K_{n-i}\right)\left((q-1)^{i-1} e_{i}(x)\right) \prod_{j=n-i+1}^{n-1}[j]_{q}
$$


The recurrence for the complete graphs in Theorem 2.4 can be found in [3, Proposition 5.18]. The theorems above are equivalent via the plethystic relation (see [8])

$$
\frac{\operatorname{LLT}(G)[(q-1) x]}{(q-1)^{n}}=\operatorname{csf}_{q}(G) .
$$

We can make this plethystic equality more explicit using Proposition 2.1. Writing

$$
L L T(G)=\sum L L T_{\lambda}(G ; q) e_{\lambda}(x)
$$

then

$$
\begin{aligned}
\omega\left(\operatorname{csf}_{q}(G)\right) & =(q-1)^{-n} \omega(\operatorname{LLT}(G)[(q-1) x]) \\
& =(q-1)^{-n} \sum \operatorname{LLT}_{\lambda}(G ; q) \omega\left(e_{\lambda}[(q-1) x]\right) \\
& =(q-1)^{-n} \sum \operatorname{LLT}_{\lambda}(G ; q) h_{\lambda}[(q-1) x] \\
& =(q-1)^{-n} \sum \operatorname{LLT}_{\lambda}(G ; q)(q-1)^{\ell(\lambda)} \rho_{\lambda}(x ; q) \\
& =\sum \operatorname{LLT}_{\lambda}(G ; q)(q-1)^{\ell(\lambda)-n} \rho_{\lambda}(x ; q),
\end{aligned}
$$

where equality (10) follows from [17, Example 1.(a), page 136] and equality (11) follows from Proposition 2.1 item (6). This proves that Theorems 1.2 and 1.3 are equivalent.

\subsection{Permutations and increasing forests}

We begin by recalling the forget cycles transformation $\sigma \rightarrow \sigma^{c}$, which simply consists of writing $\sigma$ in cycle notation and forgetting the parentheses. This means each cycle must begin with its least element, and the cycles must be ordered according to their least element. For example,

$$
\sigma=617892543, \quad \sigma=(162)(3759)(48), \quad \sigma^{c}=162375948 .
$$

Denote by $\lambda(\sigma)$ the cycle partition of $\sigma$.

For a permutation $\sigma \in S_{n}$ and Hessenberg function $\mathbf{m}:[n] \rightarrow[n]$, we define the number of $\mathbf{m}$ inversions of $\sigma$ as

$$
\operatorname{inv}_{\mathbf{m}}(\sigma):=\left|\left\{(i, j) ; i<j \leq \mathbf{m}(i), \sigma^{-1}(i)>\sigma^{-1}(j)\right\}\right| .
$$

We define the $\mathbf{m}$-weight of a permutation $\sigma$ as wt $\mathbf{m}_{\mathbf{m}}(\sigma):=\operatorname{inv}_{\mathbf{m}}\left(\sigma^{c}\right)$. We notice that $\mathrm{wt}_{\mathbf{m}}(\sigma)=\mathrm{wt}_{G_{\mathbf{m}}}(\sigma)$. Recall $\sigma \leq \mathbf{m}$ if $\sigma(i) \leq \mathbf{m}(i)$ for every $i \in[n]$, and $S_{n, \mathbf{m}}$ denotes the set $\left\{\sigma \in S_{n} ; \sigma \leq \mathbf{m}\right\}$.

Remark 2.5. We note that a $k$-cycle $\tau=\left(j_{1}, j_{2}, \ldots, j_{k}\right)$, with least element $j_{1}$, is in $S_{n, \mathbf{m}}$ if and only if $j_{l+1} \leq \mathbf{m}\left(j_{l}\right)$ for every $j \in[k-1]$. Moreover, a permutation $\sigma$ is in $S_{n, \mathbf{m}}$ if and only if all of its cycles are in $S_{n, \mathbf{m}}$ as well. Moreover, we have that the cycle $\tau \in S_{n, \mathbf{m}}$ on $\left\{j_{1}, \ldots, j_{k}\right\}$ is determined by the numbers

$$
\operatorname{inv}_{\mathbf{m}}\left(\tau, j_{l}\right)=\left|\left\{l^{\prime} \in[k] ; l^{\prime}>l, j_{l^{\prime}}<j_{l} \leq \mathbf{m}\left(j_{l}^{\prime}\right)\right\}\right|,
$$

for every $l \in[k]$ (see [11] or [3, Section 9.5]).

Now we define the weight of an increasing spanning forest of an indifference graph $G$. Before doing that, we note that given a function $g:[n] \rightarrow[n] \cup\{0\}$ such that $g(j)<j$, we have that the graph $F$ with vertex set $V(F)=[n]$ and edge set $E(F)=\{\{g(j), j\} ; g(j) \neq 0\}$ is an increasing forest. All increasing forests with vertex set $[n]$ can be obtained in this way. Given an increasing tree $T$, an edge $\{u, v\}$ with $u<v$ has a length given by $|\{w \in V(T) ; u<w<v\}|$, and we define the weight $\operatorname{wt}(T)$ of $T$ as the sum of the lengths of each edge. 

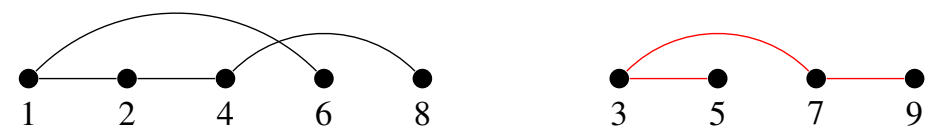

Figure 5. Computing the weight and set of inversions of the increasing forest in Figure 3.

Let $F=T_{1} \cup T_{2} \cup \ldots \cup T_{k}$ be an increasing spanning forest of a graph $G$. By convention, we assume that $\operatorname{root}\left(T_{1}\right)<\operatorname{root}\left(T_{2}\right)<\ldots<\operatorname{root}\left(T_{k}\right)$. We say that a set $\{v, w\}$ of vertices is an inversion of $F$ if $v<w$ and there exists $i<j$ such that $v \in T_{j}$ and $w \in T_{i}$. Moreover, if $\{v, w\}$ is an edge of $G$, we say

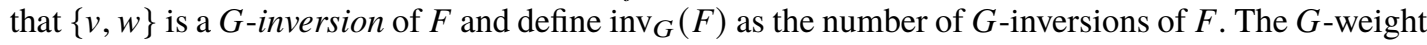
of $F$ is given by

$$
\mathrm{wt}_{G}(F):=\operatorname{inv}_{G}(F)+\sum_{1 \leq j \leq k} \operatorname{wt}\left(T_{j}\right) .
$$

When $G$ is the graph induced by a Hessenberg function $\mathbf{m}$, we write $\mathrm{wt}_{\mathbf{m}}(F)$ for $\mathrm{wt}_{G}(F)$. For a forest $F$, we say that the partition of $F$ is the partition given by $\left|V\left(T_{i}\right)\right|$ and denote it by $\lambda(F)$. For a graph $G$, we denote by $\mathcal{F}(G)$ the set of increasing spanning forests of $G$.

For example, considering the increasing forest in Figure 3, we have that $\operatorname{wt}\left(T_{1}\right)=3, \operatorname{wt}\left(T_{2}\right)=1$ and the set of inversions of $F$ is (see Figure 5)

$$
\{\{3,4\},\{3,6\},\{3,8\},\{5,6\},\{5,8\},\{7,8\}\} .
$$

The bijection between $S_{n, \mathbf{m}}$ and $\mathcal{F}\left(G_{\mathbf{m}}\right)$ given in the introduction preserves partitions, but not weights. This is solved by the proposition below.

Proposition 2.6. Given a Hessenberg function $\mathbf{m}:[n] \rightarrow[n]$, there exists a bijecion $S_{n, \mathbf{m}} \rightarrow \mathcal{F}\left(G_{\mathbf{m}}\right)$ that preserves both weight and partition.

Proof. For each cycle $\tau=\left(j_{1}, \ldots, j_{k}\right)$ in $S_{n, \mathbf{m}}$, the idea is to construct a tree $T_{\tau}$ with vertex set $\left\{j_{1}, \ldots, j_{k}\right\}$ such that $\operatorname{wt}(T)=\operatorname{inv}_{\mathbf{m}}\left(\tau^{c}\right)$. We already know it is sufficient to define a function $g:\left\{j_{2}, \ldots, j_{k}\right\} \rightarrow\left\{j_{1}, \ldots, j_{k}\right\}$ satisfying $g\left(j_{l}\right)<j_{l}$ for every $l \in\{2, \ldots, k\}$. In order to have $\operatorname{wt}\left(T_{\tau}\right)=\operatorname{inv}_{\mathbf{m}}\left(\tau^{c}\right)$, we define $g$ as the unique function satisfying

$$
\left|\left\{l^{\prime} \in[k], g\left(j_{l}\right)<j_{l^{\prime}}<j_{l}\right\}\right|=\left|\left\{l^{\prime} \in[k] ; l^{\prime}>l, j_{l^{\prime}}<j_{l} \leq \mathbf{m}\left(j_{l}^{\prime}\right)\right\}\right|
$$

for every $l \in\{2, \ldots, k\}$.

To see that $T_{\tau}$ is indeed a subgraph of $G_{\mathbf{m}}$, it is sufficient to check that $\mathbf{m}\left(g\left(j_{l}\right)\right) \geq j_{l}$ for every $l \in\{2, \ldots, k\}$. Assume for contradiction that there exists $l \in\{2, \ldots, k\}$ such that $\mathbf{m}\left(g\left(j_{l}\right)\right)<j_{l}$, so in particular we have the following inclusions:

$$
\begin{gathered}
\left\{l^{\prime} \in[k] ; g\left(j_{l}\right)<j_{l^{\prime}}<j_{l}\right\} \\
\cup \\
\left\{l^{\prime} \in[k] ; j l^{\prime}<j_{l} \leq \mathbf{m}\left(j_{l^{\prime}}\right)\right\} \\
\cup \\
\left\{l^{\prime} \in[k] ; l^{\prime}>l, j_{l^{\prime}}<j_{l} \leq \mathbf{m}\left(j_{l}^{\prime}\right)\right\} .
\end{gathered}
$$

Since $\left\{l^{\prime} \in[k], g\left(j_{l}\right)<j_{l^{\prime}}<j_{l}\right\}$ and $\left\{l^{\prime} \in[k] ; l^{\prime}>l, j_{l^{\prime}}<j_{l} \leq \mathbf{m}\left(j_{l}^{\prime}\right)\right\}$ have the same cardinality, we must have equalities in the equation above. However, since $\tau \in S_{n, \mathbf{m}}$, we have that there must exist $l^{\prime}<l$ such that $j_{l^{\prime}}<j_{l} \leq \mathbf{m}\left(j_{l^{\prime}}\right)$, which means the last inclusion is proper: a contradiction.

Let $\phi: S_{n, \mathbf{m}} \rightarrow \mathcal{F}\left(G_{\mathbf{m}}\right)$ be the function defined by $\phi(\sigma)=T_{\tau_{1}} \cup T_{\tau_{2}} \cup \ldots \cup T_{\tau_{k}}$, where $\tau_{1}, \ldots, \tau_{k}$ are the cycles of $\sigma$. The function $\phi$ is injective by Remark 2.5, and hence bijective because both sets have the same cardinality $\prod_{i=1}^{n}(\mathbf{m}(i)-i+1)$, and preserves weight and partition by construction. 


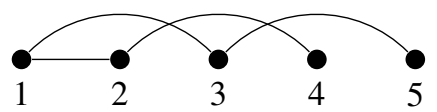

Figure 6. The increasing tree induced by the cycle $(13542) \in S_{5, \mathbf{m}}$, where $\mathbf{m}=(3,3,5,5,5)$.
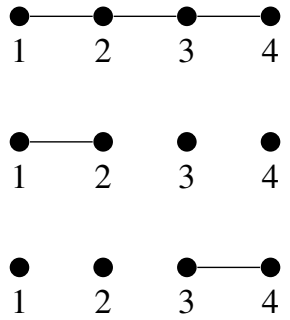
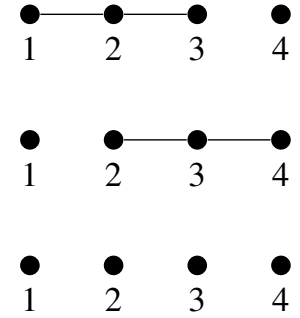
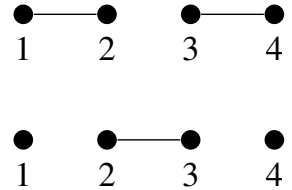

Figure 7. All the increasing spanning forests of $G_{\mathbf{m}}$ for $\mathbf{m}=(2,3,4,4)$.

We denote the increasing forest associated with $\sigma$ via this construction by $F_{\sigma, \mathbf{m}}$. For example, if $\mathbf{m}=(3,3,5,5,5)$ and $\sigma=(13542)$, then $F_{\sigma, \mathbf{m}}$ is depicted in Figure 6.

\section{The symmetric function of increasing forests}

In this section, we define a symmetric function IF: $\mathcal{D} \rightarrow \Lambda_{q}$ that enumerates increasing spanning forests of indifference graphs. First, we choose generators $y_{n}(x ; q) \in \Lambda_{q}$ homogeneous of degree $n$ and let $y_{\lambda}(x ; q)=\prod y_{\lambda_{i}}(x ; q)$. These generators will be either $y_{n}(x ; q)=\rho_{n}(x ; q)$ or $y_{n}(x ; q)=$ $(q-1)^{n-1} e_{n}(x)$ in the next section.

Definition 3.1. Let $\mathbf{m}$ be a Hessenberg function and $G_{\mathbf{m}}$ its associated graph. We define the following symmetric function (recall definition 1.1):

$$
\begin{aligned}
\mathrm{IF}(\mathbf{m})=\mathrm{IF}_{y}(\mathbf{m}) & :=\sum_{\sigma \leq \mathbf{m}} q^{\mathrm{wt}_{\mathbf{m}}(\sigma)} y_{\lambda(\sigma)}(x ; q) \\
& =\sum_{F \in \mathcal{F}\left(G_{\mathbf{m}}\right)} q^{\mathrm{wt}_{\mathbf{m}}(F)} y_{\lambda(F)}(x ; q) \\
& =\sum_{\lambda \vdash n} c_{\lambda}(\mathbf{m} ; q) y_{\lambda}(x ; q) .
\end{aligned}
$$

Example 3.2. As an example, if $\mathbf{m}=(2,3,4,4)$, we have 8 increasing spanning forests (depicted in Figure 7) of $G_{\mathbf{m}}$, all of which have weight 1 . We get

$$
\operatorname{IF}(\mathbf{m})=y_{1,1,1,1}(x ; q)+3 y_{2,1,1}(x ; q)+y_{2,2}(x ; q)+2 y_{3,1}(x ; q)+y_{4}(x ; q) .
$$

More generally, if $\mathbf{m}=(2,3, \ldots, n-1, n, n)$ is the Hessenberg function associated with the path graph, we have that the weight of any increasing spanning forest of $G_{\mathbf{m}}$ is 1 .

On the other hand, if $\mathbf{m}=(2,4,4,4)$, we have that $G_{\mathbf{m}}$ has 12 increasing spanning forests, 4 of which have partition $(3,1)$ and are depicted in Figure 8 . We get

$$
\operatorname{IF}(\mathbf{m})=y_{1,1,1,1}(x ; q)+(q+3) y_{2,1,1}(x ; q)+y_{2,2}(x ; q)+(2 q+2) y_{3,1}(x ; q)+(q+1) y_{4}(x ; q) .
$$

When $\mathbf{m}=(3,4,4,5,5)$ there are 8 increasing spanning forests of $G_{\mathbf{m}}$ with partition $(4,1)$, as depicted in Figure 9. We have 

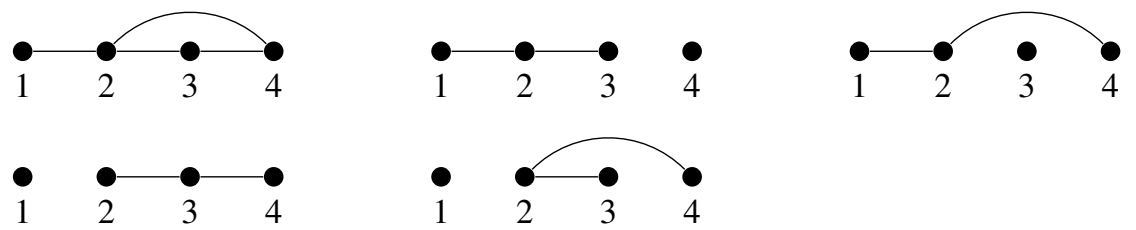

Figure 8. The graph associated with $\mathbf{m}=(2,4,4,4)$ and the increasing spanning forests of $G_{\mathbf{m}}$ with partition $(3,1)$.
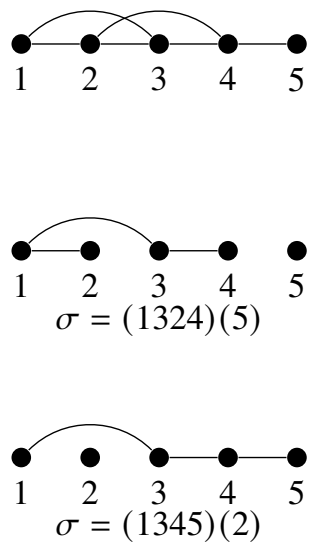
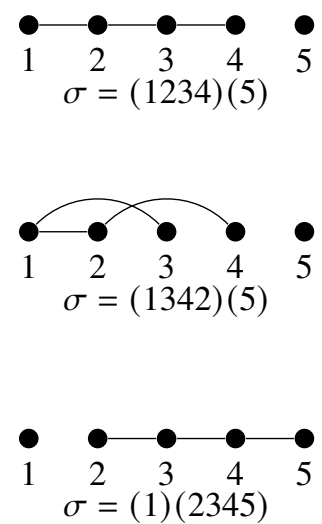
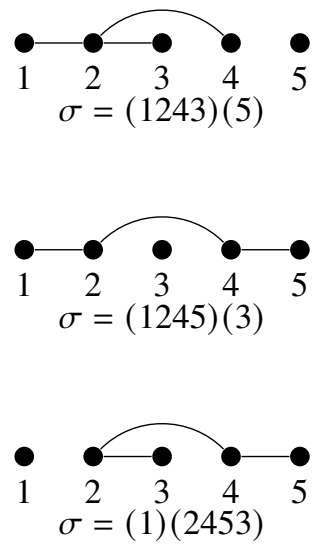

Figure 9. The graph associated with $\mathbf{m}=(3,4,4,5,5)$ and the 8 increasing spanning forests of $G_{\mathbf{m}}$ with partition $(4,1)$, with their respective permutations.

$$
\begin{aligned}
\operatorname{IF}(\mathbf{m})= & y_{1,1,1,1,1}(x ; q)+(2 q+4) y_{2,1,1,1}(x ; q)+(2 q+3) y_{2,2,1}(x ; q) \\
& +\left(q^{2}+4 q+3\right) y_{3,1,1}(x ; q)+(2 q+2) y_{3,2}(x ; q) \\
& +\left(2 q^{2}+4 q+2\right) y_{4,1}(x ; q)+\left(q^{2}+2 q+1\right) y_{5}(x ; q) .
\end{aligned}
$$

The rest of this section is devoted to proving that IF is multiplicative and satisfies the modular law, and to finding a recurrence for the values $\operatorname{IF}\left(K_{n}\right)$ at complete graphs.

Proposition 3.3. The function IF is multiplicative.

Proof. Let $G=G_{1} \cup G_{2}$ be the ordered disjoint union of $G_{1}$ and $G_{2}$. Clearly, every increasing spanning forest $F$ of $G$ is a union $F_{1} \cup F_{2}$, where $F_{i}$ is an increasing spanning forest of $G_{i}$ for $i=1,2$. Conversely, every such union $F_{1} \cup F_{2}$ is an increasing spanning forest of $G$. Moreover, we have that $\mathrm{wt}_{G}(F)=\mathrm{wt}_{G_{1}}\left(F_{1}\right)+\mathrm{wt}_{G_{2}}\left(F_{2}\right)$ and $y_{\lambda(F)}=y_{\lambda\left(F_{1}\right)} y_{\lambda\left(F_{2}\right)}$, hence

$$
\begin{aligned}
\operatorname{IF}(G) & =\sum_{F \in \mathcal{F}(G)} q^{\mathrm{wt}_{G}(F)} y_{\lambda(F)}(x ; q) \\
& =\sum_{F_{1} \in \mathcal{F}\left(G_{1}\right)} \sum_{F_{2} \in \mathcal{F}\left(G_{2}\right)} q^{\mathrm{wt}_{G_{1}}\left(F_{1}\right)+\mathrm{wt}_{G_{2}}\left(F_{2}\right)} y_{\lambda\left(F_{1}\right)}(x ; q) y_{\lambda\left(F_{2}\right)}(x ; q) \\
& =\left(\sum_{F_{1} \in \mathcal{F}\left(G_{1}\right)} q^{\mathrm{wt}_{G_{1}}\left(F_{1}\right)} y_{\lambda\left(F_{1}\right)}(x ; q)\right)\left(\sum_{F_{2} \in \mathcal{F}\left(G_{2}\right)} q^{\mathrm{wt}_{G_{2}}\left(F_{2}\right)} y_{\lambda\left(F_{2}\right)}(x ; q)\right) \\
& =\operatorname{IF}\left(G_{1}\right) \operatorname{IF}\left(G_{2}\right) .
\end{aligned}
$$

This finishes the proof. 
Table 1. The contributions of $i, j, j+1$ to the weights of $\sigma$ and $\sigma^{\prime}$ when $\sigma \leq \mathbf{m}_{0}$.

\begin{tabular}{lcccc}
\hline & $\mathrm{wt}_{\mathbf{m}_{1}}(\sigma)$ & $\mathrm{wt}_{\mathbf{m}_{0}}(\sigma)$ & $\mathrm{wt}_{\mathbf{m}_{2}}(\sigma)$ & $\mathrm{wt}_{\mathbf{m}_{2}}\left(\sigma^{\prime}\right)$ \\
\hline$i, j, j+1$ & 0 & 0 & 0 & \\
$i, j+1, j$ & 1 & 1 & 1 & \\
$j, i, j+1$ & 1 & 0 & & 2 \\
$j, j+1, i$ & 1 & 0 & 2 & \\
$j+1, i, j$ & 1 & 1 & & 1 \\
$j+1, i, j$ & 2 & 1 & 3 & \\
\hline
\end{tabular}

Table 2. The contributions of $i, j, j+1$ to the weights of $\sigma$ and $\sigma^{\prime}$ when $\sigma \not \leq \mathbf{m}_{0}$.

\begin{tabular}{cccc}
\hline & $\mathrm{wt}_{\mathbf{m}_{1}}(\sigma)$ & $\mathrm{wt}_{\mathbf{m}_{2}}(\sigma)$ & $\mathrm{wt}_{\mathbf{m}_{2}}\left(\sigma^{\prime}\right)$ \\
\hline$i, j, j+1$ & 0 & 0 & 1 \\
$j+1, i, j$ & 2 & 3 & 2
\end{tabular}

Table 3. The contributions of $i, i+1, j$ to the weights of $\sigma$ and $\sigma^{\prime}$ when $\sigma \leq \mathbf{m}_{0}$.

\begin{tabular}{lcccc}
\hline & $\mathrm{wt}_{\mathbf{m}_{1}}(\sigma)$ & $\mathrm{wt}_{\mathbf{m}_{0}}(\sigma)$ & $\mathrm{wt}_{\mathbf{m}_{2}}(\sigma)$ & $\mathrm{wt}_{\mathbf{m}_{2}}\left(\sigma^{\prime}\right)$ \\
\hline$i, i+1, j$ & 0 & 0 & 0 & \\
$i, j, i+1$ & 1 & 0 & & 2 \\
$i+1, i, j$ & 1 & 1 & 1 & \\
$i+1, j, i$ & 1 & 1 & & 1 \\
$j, i, i+1$ & 1 & 0 & 2 & \\
$j, i+1, i$ & 2 & 1 & 3 & \\
\hline
\end{tabular}

Proposition 3.4. The function IF satisfies the modular law.

Proof. Let $\mathbf{m}_{1}, \mathbf{m}_{0}, \mathbf{m}_{2}$, and $i$ as in Condition 1 , and let $j:=\mathbf{m}_{1}(i)$. Let $\sigma \leq \mathbf{m}_{1}$ be a permutation, and denote by $\sigma^{\prime}=(j, j+1) \sigma(j, j+1)$ the conjugation of $\sigma$ with the transposition $(j, j+1)$. In particular, $\sigma^{\prime c}=(j, j+1) \sigma^{c}$ and $\sigma^{\prime}$ have the same cycle partition as $\sigma$. Moreover, since $\mathbf{m}_{1}, \mathbf{m}_{0}, \mathbf{m}_{2}$, and $i$ satisfy Condition 1, we have that $\mathrm{wt}_{\mathbf{m}_{2}}\left(\sigma^{\prime}\right)=\mathrm{wt}_{\mathbf{m}_{2}}(\sigma) \pm 1$ depending on the relative position of $j, j+1$ in $\sigma^{c}$.

If $\sigma \leq \mathbf{m}_{0}$, then

$$
(1+q) q^{\mathrm{wt}_{\mathbf{m}_{1}}(\sigma)}=q \cdot q^{\mathrm{wt}_{\mathbf{m}_{0}}(\sigma)}+q^{\mathrm{wt}_{\mathbf{m}_{2}}(\tau)},
$$

where $\tau$ is chosen to be either $\sigma$ or $\sigma^{\prime}$ depending on the relative position of $i, j, j+1$ in $\sigma^{c}$, as shown in Table 1.

If $\sigma \not \leq \mathbf{m}_{0}$, then $\sigma(i)=j$, which means $i$ and $j$ are adjacent in $\sigma^{c}$. Then

$$
(1+q) q^{\mathrm{wt}_{\mathbf{m}_{1}}(\sigma)}=q^{\mathrm{wt}_{\mathbf{m}_{2}}(\sigma)}+q^{\mathrm{wt}_{\mathbf{m}_{2}}\left(\sigma^{\prime}\right)},
$$

as seen in Table 2. This proves that $(1+q) \operatorname{IF}\left(\mathbf{m}_{1}\right)=q \operatorname{IF}\left(\mathbf{m}_{0}\right)+\operatorname{IF}\left(\mathbf{m}_{2}\right)$.

Let $\mathbf{m}_{1}, \mathbf{m}_{0}, \mathbf{m}_{2}$, and $i$ as in Condition 2, and let $j:=\mathbf{m}_{1}(i+1)$. Let $\sigma \leq \mathbf{m}_{1}$ be a permutation, and denote by $\sigma^{\prime}=(i, i+1) \sigma(i, i+1)$. In particular $\sigma^{\prime c}=(i, i+1) \sigma^{c}$ and $\sigma^{\prime}$ and $\sigma$ have the same cycle partition. If $\sigma \leq \mathbf{m}_{0}$, then

$$
(1+q) q^{\mathrm{wt}_{\mathbf{m}_{1}}(\sigma)}=q \cdot q^{\mathrm{wt}_{\mathbf{m}_{0}}(\sigma)}+q^{\mathrm{wt}_{\mathbf{m}_{2}}(\tau)}
$$

where $\tau$ is chosen to be either $\sigma$ or $\sigma^{\prime}$ depending on the relative position of $i, i+1, j$ in $\sigma^{c}$, as shown in Table 3. 
Table 4. The contributions of $i, i+1, j$ to the weights of $\sigma$ and $\sigma^{\prime}$ when $\sigma \not \mathbf{m}_{0}$.

\begin{tabular}{cccc}
\hline & $\mathrm{wt}_{\mathbf{m}_{1}}(\sigma)$ & $\mathrm{wt}_{\mathbf{m}_{2}}(\sigma)$ & $\mathrm{wt}_{\mathbf{m}_{2}}\left(\sigma^{\prime}\right)$ \\
\hline$i, i+1, j$ & 0 & 0 & 1 \\
$i+1, j, i$ & 1 & 2 & 1 \\
\hline
\end{tabular}

If $\sigma \not \leq \mathbf{m}_{0}$, then $\sigma(i+1)=j$, which means $i+1$ and $j$ are adjacent in $\sigma^{c}$. Then

$$
(1+q) q^{\mathrm{wt}_{\mathbf{m}_{1}}(\sigma)}=q^{\mathrm{wt}_{\mathbf{m}_{2}}(\sigma)}+q^{\mathrm{wt}_{\mathbf{m}_{2}}\left(\sigma^{\prime}\right)},
$$

as seen in Table 4. This proves that $(1+q) \operatorname{IF}\left(\mathbf{m}_{1}\right)=q \operatorname{IF}\left(\mathbf{m}_{0}\right)+\operatorname{IF}\left(\mathbf{m}_{2}\right)$ and finishes the proof.

Proposition 3.5. We have the following recursion for the complete graph.

$$
\operatorname{IF}\left(K_{n}\right)=\sum_{i=1}^{n} \operatorname{IF}\left(K_{n-i}\right) y_{i}(x ; q) \prod_{j=n-i+1}^{n-1}[j]_{q}, \quad \operatorname{IF}\left(K_{0}\right)=1 .
$$

Proof. We have that

$$
\operatorname{IF}\left(K_{n}\right)=\sum_{F \in \mathcal{F}\left(K_{n}\right)} q^{\mathrm{wt}_{K_{n}}(F)} y_{\lambda(F)}(x ; q) .
$$

We split this sum by the number of vertices of the tree $T_{1}$ with root 1 . If $T_{1}$ has size $i$ and other vertices $l_{1}, \ldots, l_{i-1}$, then the contribution of this tree to the weight of each forest containing $T_{1}$ is

$$
\mathrm{wt}\left(T_{1}\right)+\sum_{j=1}^{i-1}\left(l_{j}-j-1\right)(j-1)
$$

Hence

$$
\sum_{F \in F\left(K_{n}\right), T_{1} \subset F} q^{\mathrm{wt}_{K_{n}}(F)} y_{\lambda(F)}(x ; q)=q^{\mathrm{wt}\left(T_{1}\right)+\sum_{j=1}^{i-1}\left(l_{j}-j-1\right)(j-1)} y_{i}(x ; q) \operatorname{IF}\left(K_{n-i}\right) .
$$

Varying $T_{1}$, we have that the sum $\sum_{1<l_{1}<l_{2}<\ldots<l_{i-1} \leq n} q^{\sum\left(l_{j}-j-1\right)(j-1)}$ is given by the $q$-binomial coefficient $\left(\begin{array}{c}n-1 \\ i-1\end{array}\right)_{q}$ (see [18]), while the sum of the weights of all increasing trees with vertex set $[i]$ is $(i-1) !_{q}$. Then

$$
\sum_{F \in F\left(K_{n}\right),\left|T_{1}\right|=i} q^{\mathrm{wt}_{K_{n}}(F)} y_{\lambda(F)}(x ; q)=(i-1) !_{q}\left(\begin{array}{c}
n-1 \\
i-1
\end{array}\right)_{q} \operatorname{IF}\left(K_{n-i}\right) y_{i}(x ; q)
$$

and the result follows.

For a subset $I=\left\{i_{1}, \ldots, i_{k}\right\} \subset[n-1]$, we define $\lambda(I)$ as the conjugate of the partition associated with the composition $\left(i_{1}, i_{2}-i_{1}, \ldots, i_{k}-i_{k-1}, n-i_{k}\right)$.

Proposition 3.6. We have that

$$
\operatorname{IF}\left(K_{n}\right)=\sum_{I \subset[n-1]} y_{\lambda(I)}(x ; q) \prod_{j \in I}[j]_{q} .
$$


Proof. We will proceed by induction on $n$. The base case $n=0$ is trivial. For $I \subset[n-1]$, define $I_{1}$ and $I_{2}$ as $I_{1}=I \cap[n-i-1], I_{2}=\{n-i+1, \ldots, n-1\}$ and $I=I_{1} \cup I_{2}$. Then $y_{\lambda(I)}=y_{\lambda_{I_{1}}} y_{i}$ and

$$
\begin{aligned}
\sum_{I \subset[n-1]} y_{\lambda(I)}(x ; q) \prod_{j \in I}[j]_{q} & =\sum_{i=1}^{n} \sum_{I_{1} \subset[n-i-1]} y_{\lambda\left(I_{1}\right)}(x ; q) y_{i}(x ; q) \prod_{j \in I_{1}}[j]_{q} \prod_{j=n-i+1}^{n-1}[j]_{q} \\
& =\sum_{i=1}^{n} \operatorname{IF}\left(K_{n-i}\right) y_{i}(x ; q) \prod_{j=n-i+1}^{n-i}[j]_{q} \\
& =\operatorname{IF}\left(K_{n}\right),
\end{aligned}
$$

where equality in (12) follows by the induction hypothesis and equality in (13) follows by Proposition 3.5.

We can now relate the coefficients of $\operatorname{IF}\left(K_{n}\right)$ with the $q$-Stirling numbers of the first kind $s_{q}(n, k)$. Recall the definition of $c_{\lambda}\left(K_{n}, q\right)$ in Definition 1.1 and that the weight $\mathrm{wt}_{K_{n}}(\sigma)$ is precisely the number of inversions of $\sigma^{c}$, the permutation obtained by removing the parentheses in the cyclic notation of $\sigma$.

Corollary 3.7. We have that

$$
\sum_{\lambda \vdash n, \ell(\lambda)=k} c_{\lambda}\left(K_{n}, q\right)=s_{q}(n, k) .
$$

In other words, the following equality holds:

$$
\sum_{\sigma \in S_{n}} q^{\mathrm{wt}_{K_{n}}(\sigma)} t^{\ell(\lambda(\sigma))}=\sum_{0 \leq k \leq n} s_{q}(n, k) t^{k}
$$

Proof. This follows immediately by noticing that both sides are equal to

$$
\sum_{\substack{I \subset[n-1] \\|I|=n-k}} \prod_{j \in I}[j]_{q}
$$

Finally, we compute the sum of the coefficients $c_{\lambda}(\mathbf{m}, q)$ for a given $\mathbf{m} \in \mathcal{D}$.

Proposition 3.8. For $\mathbf{m} \in \mathcal{D}$, we have that

$$
\sum_{\sigma \leq \mathbf{m}} q^{\mathrm{wt}_{\mathbf{m}}(\sigma)}=\prod_{j=1}^{n}\left(1+[\mathbf{m}(j)-j]_{q}\right) .
$$

Proof. Both sides of the equation are multiplicative and satisfy the modular law. By [1, Theorem 1.2], it is enough to prove the proposition for the complete graph. Let $k_{n}$ be the Hessenberg function associated with $K_{n}$ : that is, $k_{n}(i)=n$ for every $i \in[n]$. By Proposition 3.6, we have that

$$
\sum_{\sigma \leq k_{n}} q^{\mathrm{wt}_{\mathbf{m}}(\sigma)}=\sum_{I \subset[n-i]} \prod_{j \in I}[j]_{q}=\prod_{j=1}^{n-1}\left(1+[j]_{q}\right)=\prod_{j=1}^{n}\left(1+\left[k_{n}(j)-j\right]_{q}\right),
$$

which completes the proof.

Remark 3.9. The left-hand side of Equation (14) specializes, via $q=1$, to the number of acyclic orientations of the graph $G_{\mathbf{m}}$. In fact, the analogous equation for acyclic orientation reads (see [3, 
Corollary 9.3])

$$
\sum_{\theta \in A O\left(G_{\mathbf{m}}\right)} q^{\operatorname{asc}_{\mathbf{m}}(\theta)}=\prod_{i=1}^{n}[\mathbf{m}(i)-i+1]_{q},
$$

where $G_{\mathbf{m}}$ is the indifference graph associated, $A O\left(G_{\mathbf{m}}\right)$ is the set of acyclic orientations of $G_{\mathbf{m}}$ and $\operatorname{asc}_{\mathbf{m}}(\theta)$ is the number of ascending edges of $\theta$ (oriented edges $i \rightarrow j$ with $i<j$ ).

\section{Relation with the chromatic quasisymmetric function and LLT polynomials}

In this section, we prove Theorems 1.2 and 1.3.

Proof of Theorem 1.2. We begin by noticing that the right-hand side of Equation 5 is precisely $\operatorname{IF}_{\rho}(\mathbf{m})$. Since both sides $\operatorname{csf}_{q}$ and IF are multiplicative and satisfy the modular law (by Theorem 2.3, Proposition 3.3 and Proposition 3.4), it is sufficient to prove the equality in the case of complete graphs. By Proposition 3.6, we have that

$$
\operatorname{IF}_{\rho}\left(K_{n}\right)=\left|\begin{array}{cccccc}
\rho_{1}(x ; q) & \rho_{2}(x ; q) & \rho_{3}(x ; q) & \cdots & \rho_{n-1}(x ; q) & \rho_{n}(x ; q) \\
-1 & \rho_{1}(x ; q) & \rho_{2}(x ; q) & \cdots & \rho_{n-2}(x ; q) & \rho_{n-1}(x ; q) \\
0 & -[2]_{q} & \rho_{1}(x ; q) & \cdots & \rho_{n-3}(x ; q) & \rho_{n-2}(x ; q) \\
0 & 0 & -[3]_{q} & \cdots & \rho_{n-4}(x ; q) & \rho_{n-3}(x ; q) \\
\vdots & \vdots & \vdots & \ddots & \vdots & \vdots \\
0 & 0 & 0 & \cdots & -[n-1]_{q} & \rho_{1}(x ; q)
\end{array}\right|,
$$

which by Proposition 2.1 means $\operatorname{IF}_{\rho}\left(K_{n}\right)=n !_{q} h_{n}(x ; q)=\omega\left(\operatorname{csf}_{q}\left(K_{n}\right)\right)$.

Proof of Theorem 1.3. If we take $y_{n}(x ; q)=(q-1)^{n-1} e_{n}(x)$, then the right-hand side of Equation (6) is precisely $\operatorname{IF}_{y}(G)$. By Theorem 2.4, Proposition 3.3, Proposition 3.4, and Proposition 3.5, we have that both LLT and $\mathrm{IF}_{y}$ are multiplicative, satisfy the modular law, and have the same recurrence for complete graphs. This means LLT $=\mathrm{IF}_{y}$, and the result follows.

We now relate increasing spanning forests of an indifference graph $G$ to orientations of $G$, following [2] and [4]. We say that an oriented edge $\overrightarrow{u v}$ of $G$ is oriented to the right if $u<v$ and oriented to the left otherwise. Given an orientation $o$ of $G$, we remove all edges that are oriented to the right and consider the function $\operatorname{lrv}_{o}:[n] \rightarrow[n]$ (called the lowest reaching vertex), where $\operatorname{lrv}(v)$ is the minimum $u$ that is reachable from $v$. Consider the partition $\lambda(o)$ of $o$ as the partition induced by $\left|\operatorname{lrv}^{-1}(i)\right|$ for $i \in[n]$. Moreover, to each orientation $o$, we define the weight wt $(o)$ as the number of edges oriented to the left.

To give a precise meaning to this relation, we construct a function

$$
g: \mathcal{O}\left(G_{\mathbf{m}}\right) \rightarrow \mathcal{F}\left(G_{\mathbf{m}}\right)
$$

in the following way. Let $o \in \mathcal{O}\left(G_{\mathbf{m}}\right)$ be an orientation. Remove all edges that are oriented to the right and all edges $\overrightarrow{v w}$ that are oriented to the left such that $\operatorname{lrv}_{o}(v)<\operatorname{lrv}_{o}(w)$ (this means no path from $v$ to its lowest-reaching vertex goes through the edge $\overrightarrow{v w}$ ). Now, for each vertex $v$, choose (if it exists) the edge with source $v$ and with greatest length. Define the forest $g(o)$ as the forest induced by the chosen edges. Clearly we have that $\lambda(o)=\lambda(g(o))$.

We will now give another proof of [4, Corollary 2.10] for indifference graphs.

Proposition 4.1. For each $F \in \mathcal{F}\left(G_{\mathbf{m}}\right)$, the following equality holds:

$$
\sum_{o \in g^{-1}(F)} q^{\mathrm{wt}(o)}=q^{(n-\ell(\lambda(F)))}(q+1)^{\mathrm{wt}_{\mathbf{m}}(F)} .
$$



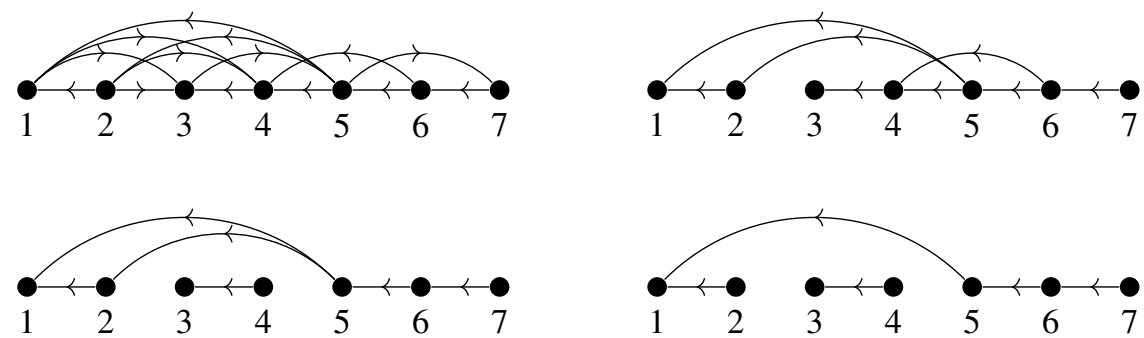

Figure 10. From an orientation to an increasing tree. At top right, we remove all edges oriented to the right. At bottom left, we remove all edges oriented to the left that do not reach the lowest-reaching vertex. At bottom right, we choose the edges with greatest length.
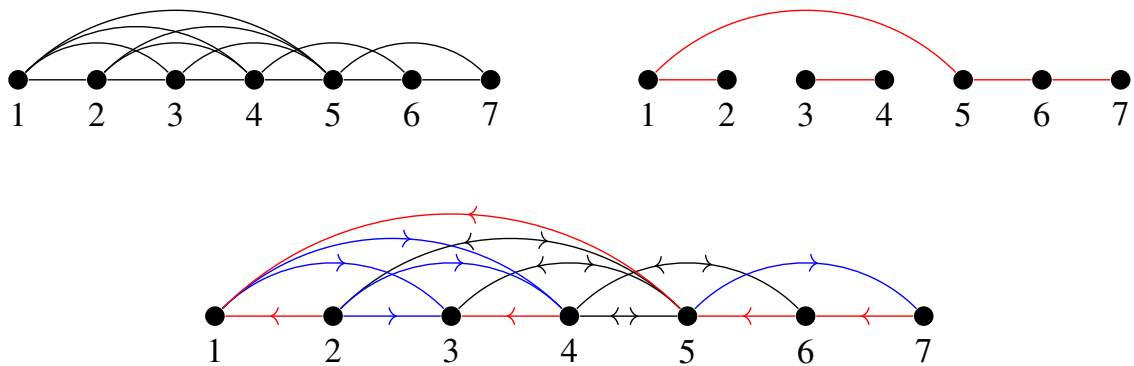

Figure 11. An indifference graph $G$, an increasing forest $F$ of $G$ and the orientations $o \in g^{-1}(G)$.

In particular, if $G$ is an indifference graph, then

$$
\operatorname{LLT}(G, q+1)=\sum_{o \in \mathcal{O}(G)} q^{\mathrm{wt}(o)} e_{\lambda(o)}(x)
$$

Proof. Let us prove the first statement. For $o \in g^{-1}(F)$, each edge in $F$ must be oriented to the left (in Figure 11, these are the edges in red), which contributes with $q^{n-\ell(\lambda(F))}$ to the weight of $o$. Each edge that connects two components of $F$ and that is not an inversion must be oriented to the right (in Figure 11 , these are the edges $\{1,3\},\{1,4\},\{2,3\}$, and $\{2,4\})$. These edges do not contribute to the weight. Each edge that connects two components of $F$ and that is an inversion can be oriented either way (in Figure 11, these are the edges $\{3,5\},\{4,5\}$ and $\{4,6\})$. These edges contribute with $(q+1)^{\operatorname{inv}(F)}$ to the sum of the weights. The edges $\{u, v\}$, with $u<v$, that are not in $F$ but connect the same component $T$ in $F$ either have to be oriented right, if $\left\{u^{\prime}, v\right\} \in T$ for some $u<u^{\prime}<v$ (in Figure 11, this is the edge $\{5,7\}$ ) or can be oriented either way (in Figure 11, this is the edge $\{2,5\}$ ). These edges contribute with $(q+1)^{\mathrm{wt}(T)}$ to the sum of the weights.

By Theorem 1.3, we have that

$$
\operatorname{LLT}\left(G_{\mathbf{m}} ; q+1\right)=\sum_{F \in F\left(G_{\mathbf{m}}\right)} q^{n-\ell(\lambda(F))}(q+1)^{\mathrm{wt}_{\mathbf{m}}(F)} e_{\lambda(F)}(x),
$$

from which the second statement follows.

We finish this section with a few comments about the vertical strip LLT polynomials. We refer the reader to [16] for the original definition and to [3] for the definition given here.

Given a Hessenberg function $\mathbf{m}:[n] \rightarrow[n]$, we say that a set $S \subset\{n-1\}$ is a decoration on $\mathbf{m}$ if $\mathbf{m}(i)>\max \{\mathbf{m}(i-1), i\}$ (recall that we set $\mathbf{m}(0)=0$ ) for every $i \in S$. We define $\operatorname{LLT}(\mathbf{m}, S)$ 
and $\operatorname{IF}_{y}(\mathbf{m}, S)$ by the following recursive formulas. If $S=\emptyset$, then $\operatorname{LLT}(\mathbf{m}, S)=\operatorname{LLT}(\mathbf{m})$ and $\operatorname{IF}_{y}(\mathbf{m}, S)=\operatorname{IF}_{y}(\mathbf{m})$; otherwise, for every $i \in S$, we have

$$
\begin{aligned}
\operatorname{LLT}(\mathbf{m}, S) & :=\frac{\operatorname{LLT}\left(\mathbf{m}, S^{\prime}\right)-\operatorname{LLT}\left(\mathbf{m}^{\prime}, S^{\prime}\right)}{q-1} \\
\operatorname{IF}_{y}(\mathbf{m}, S) & :=\operatorname{IF}_{y}\left(\mathbf{m}, S^{\prime}\right)-\operatorname{IF}_{y}\left(\mathbf{m}^{\prime}, S^{\prime}\right),
\end{aligned}
$$

where $S^{\prime}=S \backslash\{i\}$ and

$$
\mathbf{m}^{\prime}(j)= \begin{cases}\mathbf{m}(j) & \text { if } j \neq i \\ \mathbf{m}(i)-1 & \text { if } j=i\end{cases}
$$

In particular, if $y_{n}(x ; q)=(q-1)^{n-1} e_{n}(x)$ then, by Theorem 1.3, we have that LLT $(\mathbf{m}, S)=$ $\operatorname{IF}_{y}(\mathbf{m}, S) /(q-1)^{|S|}$. As in Definition 3.1 we write

$$
\operatorname{IF}_{y}(\mathbf{m}, S)=\sum_{\lambda \vdash n} c_{\lambda}(\mathbf{m}, S ; q) y_{\lambda}(x ; q) .
$$

Proposition 4.2. We have that $c_{\lambda}(\mathbf{m}, S ; q+1) \in \mathbb{N}[q]$. In particular, $\operatorname{LLT}(\mathbf{m}, S ; q+1)$ is e-positive.

Proof. For each subset $S^{\prime} \subset S$, define $\mathbf{m}_{S^{\prime}}$ as

$$
\mathbf{m}_{S^{\prime}}(j)= \begin{cases}\mathbf{m}(j) & \text { if } j \notin S^{\prime} \\ \mathbf{m}(j)-1 & \text { if } j \in S^{\prime}\end{cases}
$$

Then

$$
c_{\lambda}(\mathbf{m}, S)=\sum_{S^{\prime} \subset S}(-1)^{\left|S^{\prime}\right|} c_{\lambda}\left(\mathbf{m}_{S^{\prime}}\right)=\sum_{F \in F\left(G_{\mathbf{m}}\right)} \sum_{S^{\prime} \subset S}(-1)^{\left|S^{\prime}\right|} q^{\mathrm{wt}_{\mathbf{m}_{S^{\prime}}}(F)}
$$

where we assume that $q^{\mathrm{wt}_{\mathbf{m}_{S^{\prime}}}(F)}=0$ if $F \notin F\left(G_{\mathbf{m}_{S^{\prime}}}\right)$. However, for an increasing forest $F$, if there exists $i \in S$ such that the edge $(i, \mathbf{m}(i))$ is neither an edge of $F$ nor an inversion of $F$, then $q^{\mathrm{wt}_{\mathbf{m}^{\prime}}}(F)=$

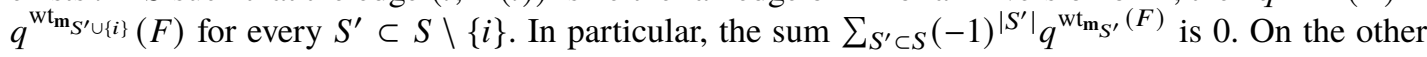
hand, if the edge $(i, \mathbf{m}(i))$ is either an edge of $F$ or an inversion of $F$ for every $i \in S$, then

$$
\sum_{S^{\prime} \subset S}(-1)^{\left|S^{\prime}\right|} q^{\mathrm{wt}_{\mathbf{m}^{\prime}}}(F)=q^{r}(q-1)^{k}
$$

where $k=\left|S_{F}\right|$ and $r=\mathrm{wt}_{\mathbf{m}_{S_{F}}}(F)$ for $S_{F}=\{i \in S ;(i, \mathbf{m}(i))$ is a inversion of $F\}$. This finishes the proof.

The combinatorial description of $\operatorname{LLT}(G, q+1)$ given in Proposition 4.1 has been recently generalized to LLT-cumulants (see [15]).

Remark 4.3. It is not true in general that $c_{\lambda}(\mathbf{m}, S ; q) \in \mathbb{N}[q]$. For example, for $\mathbf{m}=(4,5,5,5,5)$ and $S=\{1,2\}$, we have that

$$
\begin{aligned}
\operatorname{IF}(\mathbf{m}, S)= & q^{3} y_{2,2,1}(x ; q)+\left(q^{4}-q^{3}\right) y_{3,1,1}(x ; q)+\left(q^{4}+q^{3}\right) y_{3,2}(x ; q) \\
& +\left(q^{5}+q^{4}-q^{3}\right) y_{4,1}(x ; q)+\left(q^{5}+q^{4}\right) y_{5}(x ; q) .
\end{aligned}
$$

\section{Chromatic quasisymmetric functions and LLT polynomials in other bases}

In this section, we relate Theorems 1.2 and 1.3 with the known formulas for the chromatic quasisymmetric function and the LLT polynomials in other bases of $\Lambda_{q}$. 
We have already seen in Equation (3) how to compute the power-sum expansion of the chromatic symmetric function of indifference graphs. For the chromatic quasisymmetric function and the LLT polynomial, the power-sum expansions are computed in [5] and [3, Theorem 6.3], respectively. Both use the same basic objects, which we now introduce (following [3]).

Given a permutation $\sigma \in S_{n}$, a partition $\lambda \vdash n$ and a Hessenberg function $\mathbf{m}:[n] \rightarrow[n]$, we say that $\sigma$ is $(\mathbf{m}, \lambda)$-admissible if the following holds. For every $k \in\{0, \ldots, \ell(\lambda)-1\}$ and for every $i$ such that

$$
\lambda_{1}+\lambda_{2}+\ldots+\lambda_{k}<i<\lambda_{1}+\lambda_{2}+\ldots+\lambda_{k+1}
$$

we have that

$\circ \sigma\left(\lambda_{1}+\lambda_{2}+\ldots+\lambda_{k}+1\right)>\sigma(i+1)$

$\circ \sigma(i)>\sigma(i+1)$ only if $\sigma(i) \leq \mathbf{m}(\sigma(i+1))$.

If $\mathcal{N}_{\mathbf{m}, \lambda}$ is the set of $(\mathbf{m}, \lambda)$-admissible permutations, then

$$
\omega\left(\operatorname{csf}_{q}(\mathbf{m})\right)=\sum_{\lambda \vdash n} \frac{p_{\lambda}(x)}{z_{\lambda}} \prod_{i=1}^{\ell(\lambda)}\left[\lambda_{i}\right]_{q} \sum_{\sigma \in \mathcal{N}_{\mathbf{m}, \lambda}} q^{\operatorname{asc}_{\mathbf{m}}\left(\sigma^{-1}\right)},
$$

where $\operatorname{asc}_{\mathbf{m}}\left(\sigma^{-1}\right)$ is the number of pairs $1 \leq i<j \leq n$ such that $j \leq \mathbf{m}(i)$ and $\sigma^{-1}(i)<\sigma^{-1}(j)$. For the LLT polynomials, we have that

$$
\omega(\operatorname{LLT}(\mathbf{m}))=\sum_{\lambda \vdash n} \frac{p_{\lambda}(x)}{z_{\lambda}}(q-1)^{n-\ell(\lambda)} \sum_{\sigma \in \mathcal{N}_{\mathbf{m}, \lambda}} q^{\operatorname{asc}_{\mathbf{m}}\left(\sigma^{-1}\right)} .
$$

To compute the power-sum expansion of the LLT polynomias using Theorem 1.3, we note that $\omega\left(e_{\lambda}(x)\right)=h_{\lambda}(x)$ and (see [17, Equation (6.11)])

$$
h_{\lambda}(x)=\sum L_{\mu \lambda} \frac{p_{\mu}(x)}{z_{\mu}}
$$

where

$$
L_{\mu \lambda}=\left|\left\{f:[\ell(\mu)] \rightarrow \mathbb{Z}_{>0} ; \sum_{j \in f^{-1}(i)} \mu_{j}=\lambda_{i}\right\}\right|
$$

Then the power-sum expansion is

$$
\omega(\operatorname{LLT}(\mathbf{m}))=\sum_{\mu \vdash n} \frac{p_{\mu}(x)}{z_{\mu}} \sum_{F \in \mathcal{F}(G)}(q-1)^{n-\lambda(F)} q^{\mathrm{wt}_{\mathbf{m}}(F)} L_{\mu, \lambda(F)} .
$$

In particular, we have that Theorem 1.3 is equivalent to the following identity involving increasing forests and admissible permutations:

$$
\sum_{F \in \mathcal{F}\left(G_{\mathbf{m}}\right)}(q-1)^{\ell(\mu)-\ell(\lambda(F))} q^{\mathrm{wt}_{\mathbf{m}}(F)} L_{\mu, \lambda(F)}=\sum_{\sigma \in \mathcal{N}_{\mathbf{m}, \mu}} q^{\text {asc }_{\mathbf{m}}\left(\sigma^{-1}\right)} .
$$

To compute the power-sum expansion of the chromatic symmetric function, we could apply Proposition 2.1 item (7). Alternatively, it is enough to substitute the equality in Equation (16) into Equation (15).

The Schur expansion of the chromatic quasisymmetric function is already computed in [20], generalizing the work in [12]. The Schur expansion of $\rho_{\lambda}(x ; q)$ involves irreducible characters of Hecke algebras (see [19]), similar to how the Schur expansion of $p_{n}$ involves characters of the symmetric group. We will not delve into this subject and leave it to the reader familiar with these topics to deduce the induced equalities. 
The LLT polynomial is known to be Schur-positive, but no combinatorial proof is known at the time. Equation (7), when expanded in the Schur basis, becomes

$$
\operatorname{LLT}(\mathbf{m})=\sum_{\mu \vdash n} s_{\mu}(x)\left(\sum_{F \in \mathcal{F}(G)}(q-1)^{n-\ell(\lambda(F))} q^{\mathrm{wt}_{\mathbf{m}}(F)} K_{\mu^{\prime}, \lambda(f)}\right),
$$

where $K_{\mu \lambda}$ is the Kostka coefficient and $\mu^{\prime}$ is the partition conjugated to $\mu$. This formulation is equivalent (in the unicellular case) to [2, Corollary 6.2] via Proposition 4.1.

Both Equations (16) and (17) have sums involving powers of $(q-1)$ that turn out to be polynomials in $q$ with positive coefficients. It is not clear to the authors how to give a direct proof of the equality in Equation (16) nor that the sum in Equation (17) is a polynomial with positive coefficients.

One can also use Theorem 1.2 to write the expansion of $\operatorname{csf}_{q}(G)$ in the elementary basis. By Proposition 2.1 item (5), we can express the $e$-coefficients of $\operatorname{csf}_{q}(G)$ as a $\mathbb{Q}(q)$-linear combination of $\left(c_{\mu}(G ; q)\right)_{\mu \vdash n}\left(\right.$ recall Definition 1.1) for any indifference graph $G$. Define $\operatorname{csf}_{\lambda}(G ; q)$ via

$$
\operatorname{csf}_{q}(G)=\sum_{\lambda} \operatorname{csf}_{\lambda}(G ; q) e_{\lambda}(x)
$$

We have that

$$
\operatorname{csf}_{\lambda}(G ; q)=\sum_{\mu \vdash n}(-1)^{\ell(\lambda)-\ell(\mu)} w_{\mu \lambda}(q) c_{\mu}(G ; q),
$$

where $w_{\mu \lambda}(q)$ is defined in Section 2.1. For example,

1. If $\lambda=(n)$, then $\operatorname{csf}_{n}(G ; q)=[n]_{q} c_{\lambda}(G ; q)$;

2. If $\lambda=(a, b)$, with $b<a$, then $\operatorname{csf}_{\lambda}(G ; q)=[a]_{q}[b]_{q} c_{\lambda}(G ; q)-\left([a]_{q}+\left[b_{q}\right]\right) c_{n}(G ; q)$;

3. If $\lambda=(a, a)$, then $\operatorname{csf}_{\lambda}(G ; q)=[a]_{q}^{2} c_{\lambda}(G ; q)-[a]_{q} c_{n}(G ; q)$.

4. If $\lambda$ has distinct parts $\lambda_{1}>\lambda_{2}>\ldots>\lambda_{\ell(\lambda)}$ then

$$
w_{\mu \lambda}(q)=\sum_{A} \prod_{B \in A} \sum_{j \in B}\left[\lambda_{j}\right]_{q},
$$

where the sum runs through all ordered set partitions $A=\left(B_{1}, \ldots, B_{\ell(\mu)}\right)$ of $\{1, \ldots, \ell(\lambda)\}$ such that $\sum_{j \in B_{k}} \lambda_{j}=\mu_{k}$ for every $k=1, \ldots, \ell(\mu)$.

The Stanley-Stembridge conjecture states that $\operatorname{csf}_{\lambda}(G ; 1)$ is non-negative for every indifference graph $G$ and partition $\lambda$. More generally, Shareshian-Wachs conjectured that $\operatorname{csf}_{\lambda}(G ; q)$ is a polynomial with non-negative coefficients. Even in the cases of items (2) and (3) above, these conjectures remain open.

Acknowledgement. We thank the anonymous referee for the careful reading of the paper and constructive suggestions.

Conflict of Interest: None.

\section{References}

[1] A. Abreu and A. Nigro. Chromatic symmetric functions from the modular law. J. Combin. Theory Ser. A, 180:105407, 30, 2021.

[2] P. Alexandersson. LLT polynomials, elementary symmetric functions and melting lollipops. Journal of Algebraic Combinatorics, Apr 2020.

[3] P. Alexandersson and G. Panova. LLT polynomials, chromatic quasisymmetric functions and graphs with cycles. Discrete Math., 341(12): 3453-3482, 2018.

[4] P. Alexandersson and R. Sulzgruber. A combinatorial expansion of vertical-strip LLT polynomials in the basis of elementary symmetric functions, Preprint, 2020, arXiv:2004.09198.

[5] C.A. Athanasiadis. Power sum expansion of chromatic quasisymmetric functions. Electron. J. Combin., 22(2):Paper 2.7, 9, 2015. 
[6] F. Bergeron. Open questions for operators related to rectangular Catalan combinatorics. J. Comb., 8(4): 673-703, 2017.

[7] F. Bergeron, P. Flajolet, and B. Salvy. Varieties of increasing trees. In CAAP '92 (Rennes, 1992), volume $\mathbf{5 8 1}$ of Lecture Notes in Comput. Sci., pages 24-48. Springer, Berlin, 1992.

[8] E. Carlsson and A. Mellit. A proof of the shuffle conjecture. J. Amer. Math. Soc., 31(3): 661-697, 2018.

[9] M. D’Adderio. e-positivity of vertical strip LLT polynomials. J. Combin. Theory Ser. A, 172:105212, $15,2020$.

[10] A.M. Garsia, J. Haglund, D. Qiu, and M. Romero. $e$-positivity results and conjectures, Preprint, 2019, arXiv:1904.07912.

[11] A.M. Garsia and J.B. Remmel. $Q$-counting rook configurations and a formula of Frobenius. J. Combin. Theory Ser. A, 41(2): 246-275, 1986.

[12] V. Gasharov. Incomparability graphs of $(3+1)$-free posets are $s$-positive. In Proceedings of the 6th Conference on Formal Power Series and Algebraic Combinatorics (New Brunswick, NJ, 1994), volume 157, pages 193-197, 1996.

[13] H.W. Gould. The $q$-Stirling numbers of first and second kinds. Duke Math. J., 28: 281-289, 1961.

[14] J. Hallam and B. Sagan. Factoring the characteristic polynomial of a lattice. J. Combin. Theory Ser. A, 136: 39-63, 2015.

[15] M. Kowalski. LLT cumulants of unicellular Young diagrams, parking functions and Schur positivity, 2020. arxiv:2011.15080.

[16] A. Lascoux, B. Leclerc, and J.-Y. Thibon. Ribbon tableaux, Hall-Littlewood functions, quantum affine algebras, and unipotent varieties. J. Math. Phys., 38(2): 1041-1068, 1997.

[17] I.G. Macdonald. Symmetric functions and Hall polynomials. Oxford Classic Texts in the Physical Sciences. The Clarendon Press, Oxford University Press, New York, second edition, 2015. With contribution by A. V. Zelevinsky and a foreword by Richard Stanley, Reprint of the 2008 paperback edition.

[18] G. Pólya. Gaussian binomial coefficients and the enumeration of inversions. In Proc. Second Chapel Hill Conf. on Combinatorial Mathematics and its Applications (Univ. North Carolina, Chapel Hill, N.C., 1970), pages 381-384. Univ. North Carolina, Chapel Hill, N.C., 1970.

[19] A. Ram. A Frobenius formula for the characters of the Hecke algebras. Invent. Math., 106(3): 461-488, 1991.

[20] J. Shareshian and M.L. Wachs. Chromatic quasisymmetric functions. Adv. Math., 295: 497-551, 2016.

[21] R.P. Stanley. A symmetric function generalization of the chromatic polynomial of a graph. Adv. Math., 111(1): 166-194, 1995.

[22] R.P. Stanley. Enumerative combinatorics. Volume 1, volume 49 of Cambridge Studies in Advanced Mathematics. Cambridge University Press, Cambridge, second edition, 2012.

[23] R. P. Stanley and J. R. Stembridge. On immanants of Jacobi-Trudi matrices and permutations with restricted position. $J$. Combin. Theory Ser. A, 62(2): 261-279, 1993. 